\title{
Multiplex Real-Time Polymerase Chain Reaction on Sputum for the Diagnosis of Pneumocystis jirovecii Pneumonia in Children: A Retrospective Study
}

\author{
Juan Jiang ${ }^{1,2}$ \\ Xia Wang ${ }^{3}$ \\ Jian $\mathrm{He}^{3}$ \\ Donglei Liao ${ }^{3}$ \\ Xiaolu Deng (D) $^{3}$
}

'Department of Respiratory Medicine (National Key Clinical Specialty), Xiangya Hospital, Central South University, Changsha, People's Republic of China; ${ }^{2}$ Hunan Provincial Clinical Research Center for Respiratory Diseases, Changsha, People's Republic of China; ${ }^{3}$ Department of Pediatrics, Xiangya Hospital, Central South University, Changsha, People's Republic of China

Correspondence: Xiaolu Deng Department of Pediatrics, Xiangya Hospital, Central South University, Changsha, Hunan, 410008, People's

Republic of China

$\mathrm{Tel}+86$ I3786II3654

Email dengxiaolu1019@163.com
Background: Pneumocystis jirovecii pneumonia (PCP) is a serious opportunistic infection in immunocompromised children. Real-time polymerase chain reaction (PCR) is widely used for the diagnosis of PCP due to its good accuracy. However, the diagnostic performance of multiplex real-time PCR on sputum in children with PCP has never been explored.

Methods: Medical records of 63 consecutive pediatric patients were analyzed retrospectively, including 13 cases with PCP and 50 with non-PCP pneumonia. Pneumocystis jirovecii (P. jirovecii) and other co-pathogens detected by multiplex real-time PCR in sputum samples were summarized. Using clinical composite diagnosis as the reference standard, we further compared the diagnostic performance of multiplex real-time PCR to combined serological markers (1,3)- $\beta$-D-glucan plus lactate dehydrogenase. Additionally, modifications of antimicrobial treatment for pediatric PCP patients after the report of multiplex real-time PCR results were reviewed.

Results: In children with PCP, nonproductive cough and shortness of breath were more common, lymphocyte count in peripheral blood was markedly lower, and serum levels of $(1,3)-\beta$-D-glucan and lactate dehydrogenase were much higher than non-PCP group. Multiplex real-time PCR reached a sensitivity of $100 \%$ in diagnosing PCP, which was better than serum (1,3)- $\beta$-D-glucan plus lactate dehydrogenase (76.9\%). Its specificity $(98.0 \%)$ significantly surpassed serum (1,3)- $\beta$-D-glucan plus lactate dehydrogenase (84.4\%). Furthermore, multiplex real-time PCR showed a good performance in identifying co-pathogens in sputum of pediatric PCP patients. Cytomegalovirus, Epstein-Barr virus and Streptococcus pneumoniae were the most common co-pathogens in these patients. Initial antimicrobial treatment was modified in $76.9 \%$ of children with PCP after the report of PCR results.

Conclusion: Multiplex real-time PCR on sputum is a diagnostic tool with good performance for the identification of $P$. jirovecii as well as co-pathogens in children with PCP. Sputum may be an alternative to bronchoalveolar lavage fluid for PCR assay in children when bronchoscopic examination is not feasible.

Keywords: immunocompromised population, children, Pneumocystis jirovecii pneumonia, multiplex real-time polymerase chain reaction, sputum

\section{Introduction}

Pneumocystis jirovecii pneumonia (PCP) is a serious opportunistic infection in immunocompromised population across all age groups. It is caused by the infection of $P$. jirovecii, a ubiquitous fungus colonized in human airway. So far, PCP remains a major cause of morbidity and mortality in immunocompromised children with or without HIV infection. ${ }^{1}$ A previous study showed that the 30-day, 60-day, and 90- 
day mortality rates in pediatric PCP patients were $20 \%$, $24 \%$, and $32 \%$, respectively. ${ }^{2}$ In a 10 -year retrospective study, Ling and colleagues reported that PCP in non-HIV children was a serious illness with a mortality as high as $41.7 \%{ }^{3}$

Rapid and accurate diagnosis of PCP in children has remained challenging. Neither clinical manifestations nor radiological features of PCP patients are specific. Bilateral diffuse ground glass opacities are suggestive but not unique to PCP. So far, P. jirovecii cannot be readily cultured in vitro. Serum $(1,3)-\beta$-D-glucan (BDG) and lactate dehydrogenase (LDH) are considered as sensitive but unspecific markers of PCP. ${ }^{4,5}$ Definite diagnosis of PCP relies on direct microscopic examination of $P$. jirovecii in respiratory tract specimen after conventional or immunofluorescence staining. But microscopic examination is insensitive and operator-dependent. ${ }^{6}$ Currently, real-time polymerase chain reaction (PCR) has been widely used for detecting $P$. jirovecii due to its good sensitivity (9499\%) and specificity (89-93\%). ${ }^{7}$ Multiplex real-time PCR is a validated strategy for the rapid detection of a number of pathogens by incorporating multiple primers within a single reaction tube to amplify genomic fragments of different pathogens. ${ }^{8}$ Compared to standard PCR, multiplex real-time PCR is less time-consuming and laborintensive, and its utility in identifying pathogens in mixed infections has been well recognized. ${ }^{8,9}$

Bronchoalveolar lavage fluid (BALF) is the most frequently used and studied respiratory tract specimen in PCR assay of $P$. jirovecii. However, as an invasive operation, bronchoalveolar lavage can be infeasible and unsafe, especially for pediatric PCP patients who usually present with serious hypoxemia. Infants and most of young children are unable to act as required by bronchoscopists during operation; thus, general anesthesia is often needed, which increases the complexity and risk of bronchoalveolar lavage. Compared to BALF, sputum samples are readily accessible, non-invasive and repeatable. It has been reported that PCR assay on sputum reached a high sensitivity of $84.62 \%$ and specificity of $98.4 \%$ for the diagnosis of PCP in immunocompromised adults. ${ }^{10} \mathrm{~A}$ previous study also suggested that sputum may be a viable alternative to bronchoscopic specimens for PCR assay of $P$. jirovecii. ${ }^{11}$ However, the utility of multiplex real-time PCR on sputum for the diagnosis of PCP in children has been largely unexplored.
Herein, we evaluated the diagnostic performance of multiplex real-time PCR in pediatric PCP patients by conducting a retrospective study.

\section{Materials and Methods}

\section{Study Design and Subjects}

This is a retrospective study. From 1 January 2019 to 31 March 2021, a total of 13 pediatric patients $(<14$ years old) were admitted to Xiangya Hospital, Central South University (Changsha, China) and diagnosed as PCP. Patients were eligible for enrolment if they met all the following criteria: (1) immunocompromised conditions, including but not limited to HIV infection, hematologic malignancies, solid tumors, long-term systemic use of corticosteroids, use of immunosuppressive agents, solid organ transplantation, hematopoietic stem cell transplantation, radiotherapy and/or chemotherapy for malignancies. (2) Typical clinical manifestations of PCP, including fever, nonproductive cough, shortness of breath, and progressive hypoxemia. (3) Radiological abnormalities suggestive to PCP in bilateral lungs revealed by chest computed tomography (CT). (4) sputum samples were collected for multiplex real-time PCR assay. Final diagnosis of PCP was made based on clinical symptoms, laboratory findings, chest radiology, microbiological tests and treatment response, by an independent committee of physicians, which including pediatricians and pulmonologists. Meanwhile, there were totally 50 non-PCP pediatric patients who were admitted to the same hospital during the same time interval because of pneumonia and had their sputum samples sent for $P$. jirovecii detection by multiplex real-time PCR. These patients were enrolled as control. Patients in control group were confirmed as non-PCP pneumonia on the basis of symptoms, clinical examinations, radiological features and microbiological tests in respiratory tract specimens and/or blood. Patients were excluded if they met any of the following criteria: (1) multiplex real-time PCR on sputum was not performed; (2) medical record was incomplete.

\section{Collection of Sputum Samples}

Sputum samples were obtained by aspirating tracheal secretions through a catheter connected to a sputum trap and fitted to a vacuum source. Briefly, a catheter was inserted into the trachea of a pediatric patient, and a mural vacuum pump was used for suction of secretions. Each sputum sample was introduced in a sterile tube using 
a sputum extractor device and sent for detection of $P$. jirovecii within 30 minutes as far as possible.

\section{Detection of $P$. jirovecii and Other Pathogens by Multiplex Real-Time PCR}

Multiplex real-time PCR was performed according to the manufacturer's instruction using Respiratory Panel Diagnostic Kit (KingMed Diagnostics, China). This kit worked based on the real-time quantitative PCRFluorescence Probing method, and the results were expressed in quantitative cycle threshold $(\mathrm{Ct})$ values. Targets of multiplex PCR were listed as follows: $P$. jirovecii, influenza A virus (H1N1, H3N2 and $\mathrm{H} 5 \mathrm{~N} 1)$, influenza $B$ virus (Yamagata and Victoria lineage), respiratory syncytial virus (A and B), human adenovirus (subtype 1, 2, 3, 4, 5, 7 and 55), human rhinovirus (A, B and C), parainfluenza virus (1, 2, 3 and 4), human cytomegalovirus, Epstein-Barr virus, human bocavirus, Mycoplasma pneumoniae, Chlamydia pneumoniae, Streptococcus pneumoniae, Staphylococcus aureus, Haemophilus influenzae, Pseudomonas aeruginosa, Legionella pneumophila, Acinetobacter baumannii, Klebsiella pneumoniae, Mycobacterium tuberculosis, Bordetella pertussis and Candida albicans. A positive result was defined as that there is a typical S-shape amplification curve, and $\mathrm{Ct} \leq 40$ by the amplification curve. On the contrary, a negative result was defined as that there is no typical $\mathrm{S}$-shape amplification curve or $\mathrm{Ct}>40$ by the amplification curve. Meanwhile, internal positive control and negative control samples were used for reaction quality control. The positive control composed of the plasmids containing target genes and internal standard gene fragments. The negative controls composed of sterile saline solution. The reaction was determined as "successful" only if there is no $\mathrm{Ct}$ value or $\mathrm{Ct}$ $>40$ for negative control, and $\mathrm{Ct} \leq 35$ for positive control. Typical amplification curves of multiplex real-time PCR are shown in Supplementary Figure 1.

\section{Statistical Analysis}

SPSS 25.0 software (IBM Corp., Armonk, NY, USA) was used for statistical analysis. Continuous variables are presented as medians and interquartile ranges, and categorical variables as counts and percentages. The Mann-Whitney $U$-test was used for comparing the differences of continuous variables between PCP and non-PCP groups, while the chi-square test or Fisher's exact test was used for categorical variables. Sensitivity, specificity, positive predict value (PPV), and negative predict value (NPV) were calculated using clinical composite diagnosis as the reference standard. Significance was fixed at $P<0.05$.

\section{Ethics Statement}

This study was approved by the Institutional Review Board and Ethics Committee of Central South University (202103115) and conducted according to the Declaration of Helsinki. All research data were de-identified and anonymously analyzed.

\section{Consent for Publication}

The informed consent was obtained from a parent or legal guardian of the patient (Case 13 of PCP group) for the images to be published.

\section{Results}

\section{Baseline Characteristics of Patients}

A total of 13 pediatric PCP patients and 50 non-PCP patients were consecutively enrolled in this study, and their baseline characteristics are shown in Table 1. No significant difference was observed in the median ages (5.7 vs 3.7 years old, $P=0.391$ ) and gender compositions ([male proportion] $61.5 \%$ vs $66.0 \%, P=0.764$ ) of two groups. The most common symptoms of pediatric PCP patients included fever $(84.6 \%)$, shortness of breath $(84.6 \%)$ and cough (69.2\%). Compared to non-PCP patients, expectoration was less frequently seen, while shortness of breath was more common in those with PCP. Systemic use of corticosteroids $(84.6 \%$ vs $24.0 \%$, $P<0.001)$, hematologic malignancies $(76.9 \%$ vs $30.0 \%$, $P=0.002)$ and use of immunosuppressive agents $(69.2 \%$ vs $20.0 \%, P=0.001)$ was significantly more common in PCP group.

The median $\mathrm{PaO} 2 / \mathrm{FiO} 2$ was $232 \mathrm{mmHg}$ in pediatric PCP patients. As expected, lymphocyte count of PCP patients was markedly lower than non-PCP group, while white blood cell and neutrophil counts of two groups were comparable. Totally, $76.9 \%$ of pediatric PCP patients showed elevated serum BDG level, and 6 out of 13 patients had serum BDG $\geq 300 \mathrm{ng} / \mathrm{L}$. Obviously, the median serum LDH level of pediatric PCP patients was remarkably higher than non-PCP group (556 vs $298 \mathrm{U} / \mathrm{L}$, $P<0.001)$.

Bilateral and multifocal involvements in lungs were seen in all pediatric PCP patients. Ground-glass opacity was revealed on chest CT images of $61.5 \%$ of PCP patients, while patchy shadowing was the most common 
Table I Baseline Clinical Characteristics, Laboratory Findings and Radiological Features of Pediatric PCP and Non-PCP Patients

\begin{tabular}{|c|c|c|c|}
\hline Characteristics (Median [IQR] or n [\%]) & PCP Group $(n=13)$ & Non-PCP Group $(n=50)$ & $P$ value \\
\hline Age (years) & $5.7(1.1-11.0)$ & $3.7(1.1-7.0)$ & 0.391 \\
\hline Male & $8(61.5)$ & $33(66.0)$ & 0.764 \\
\hline \multicolumn{4}{|l|}{ Clinical symptoms } \\
\hline Fever & II (84.6) & $32(64.0)$ & 0.155 \\
\hline Cough & $9(69.2)$ & $44(88.0)$ & 0.099 \\
\hline Expectoration & $2(I 5.4)$ & $29(58.0)$ & 0.006 \\
\hline Shortness of breath & II (84.6) & $20(40.0)$ & 0.004 \\
\hline \multicolumn{4}{|l|}{ Immunocompromised conditions } \\
\hline Hematologic malignancy & $10(76.9)$ & $15(30.0)$ & 0.002 \\
\hline Solid tumor & $0(0)$ & $2(4.0)$ & 0.464 \\
\hline AIDS & I (7.7) & $0(0)$ & 0.084 \\
\hline Systemic use of corticosteroids & II (84.6) & $12(24.0)$ & $<0.001$ \\
\hline Use of immunosuppressive agents & $9(69.2)$ & $10(20.0)$ & 0.001 \\
\hline HSC transplantation & $0(0)$ & I (2.0) & 0.607 \\
\hline $\mathrm{PaO} 2 / \mathrm{FiO} 2$ (mmHg) & $232(180-288)$ & $260(2|7-3| 3)$ & 0.63 \\
\hline WBC $\left(\times 10^{9} / \mathrm{L}\right)$ & $5.4(1.6-9.3)$ & $7.6(2.5-11.0)$ & 0.266 \\
\hline Neutrophils $\left(\times 10^{9} / \mathrm{L}\right)$ & $3.9(1.1-5.9)$ & $2.1(1.0-6.3)$ & 0.832 \\
\hline Lymphocytes $\left(\times 10^{9} / \mathrm{L}\right)$ & $0.5(0.4-1.0)$ & $2.9(0.6-4.6)$ & 0.007 \\
\hline Hemoglobin (g/L) & $93(78-106)$ & $111(94-123)$ & 0.04 \\
\hline Platelet $\left(\times 10^{9} / \mathrm{L}\right)$ & $160(117-229)$ & $265(|42-39|)$ & 0.079 \\
\hline Serum BDG (ng/L) & & $(n=32)^{*}$ & \\
\hline$\geq 80$ & $10(76.9)$ & $5(15.6)$ & $<0.001$ \\
\hline$\geq 300$ & $6(46.2)$ & $0(0)$ & $<0.001$ \\
\hline LDH (U/L) & $556(376-677)$ & $298(245-40 I)$ & $<0.001$ \\
\hline CRP (mg/L) & $14(4-47)$ & $8(2-66)$ & 0.688 \\
\hline PCT (ng/mL) & $0.27(0.08-0.60)$ & $0.29(0.05-0.89)$ & 0.979 \\
\hline \multicolumn{4}{|l|}{ Abnormalities on chest CT } \\
\hline Bilateral involvement & $13(100)$ & $35(70.0)$ & 0.027 \\
\hline Multifocal involvement & $13(100)$ & $36(72.0)$ & 0.055 \\
\hline Ground-glass opacity & $8(61.5)$ & $10(20.0)$ & 0.003 \\
\hline Patchy shadowing & $6(46.2)$ & $39(78.0)$ & 0.024 \\
\hline Consolidation & I (7.7) & $8(16.0)$ & 0.446 \\
\hline Interstitial patterns & $5(38.5)$ & $2(4.0)$ & 0.003 \\
\hline Pleural effusion & $0(0)$ & II (22.0) & 0.1 \\
\hline
\end{tabular}

Note: $(n=32)^{*}$, serum BDG was detected in 32 out of 50 non-PCP pediatric patients.

Abbreviations: IQR, interquartile range; AIDS, Acquired Immune Deficiency Syndrome; HSC, hematopoietic stem cells; PaO2, arterial partial pressure of oxygen; FiO2, fraction of inspired oxygen; BDG, (I,3)- $\beta$-D-glucan; LDH, lactate dehydrogenase; CRP, C-reactive protein; PCT, procalcitonin; CT, computed tomography.

radiological abnormality of non-PCP cohort. Consolidation and pleural effusion were uncommon in PCP. Representative chest CT images of a male pediatric PCP patient are shown in Figure 1 to present typical radiological features of PCP patients. Diffuse groundglass opacities in bilateral lungs were observed on admission (Figure 1A). After treatment, the majority of bilateral pulmonary lesions were significantly absorbed before his discharge (Figure 1B).
Multiplex Real-Time PCR Results on Sputum Samples of Pediatric PCP Patients

Table 2 shows the results of multiplex real-time PCR on sputum samples of 13 pediatric PCP patients. Generally, it took one day from sputum sampling to report. Copathogens were identified in 12 out of 13 patients. Among them, cytomegalovirus, Epstein-Barr virus and Streptococcus pneumoniae were the most common co- 

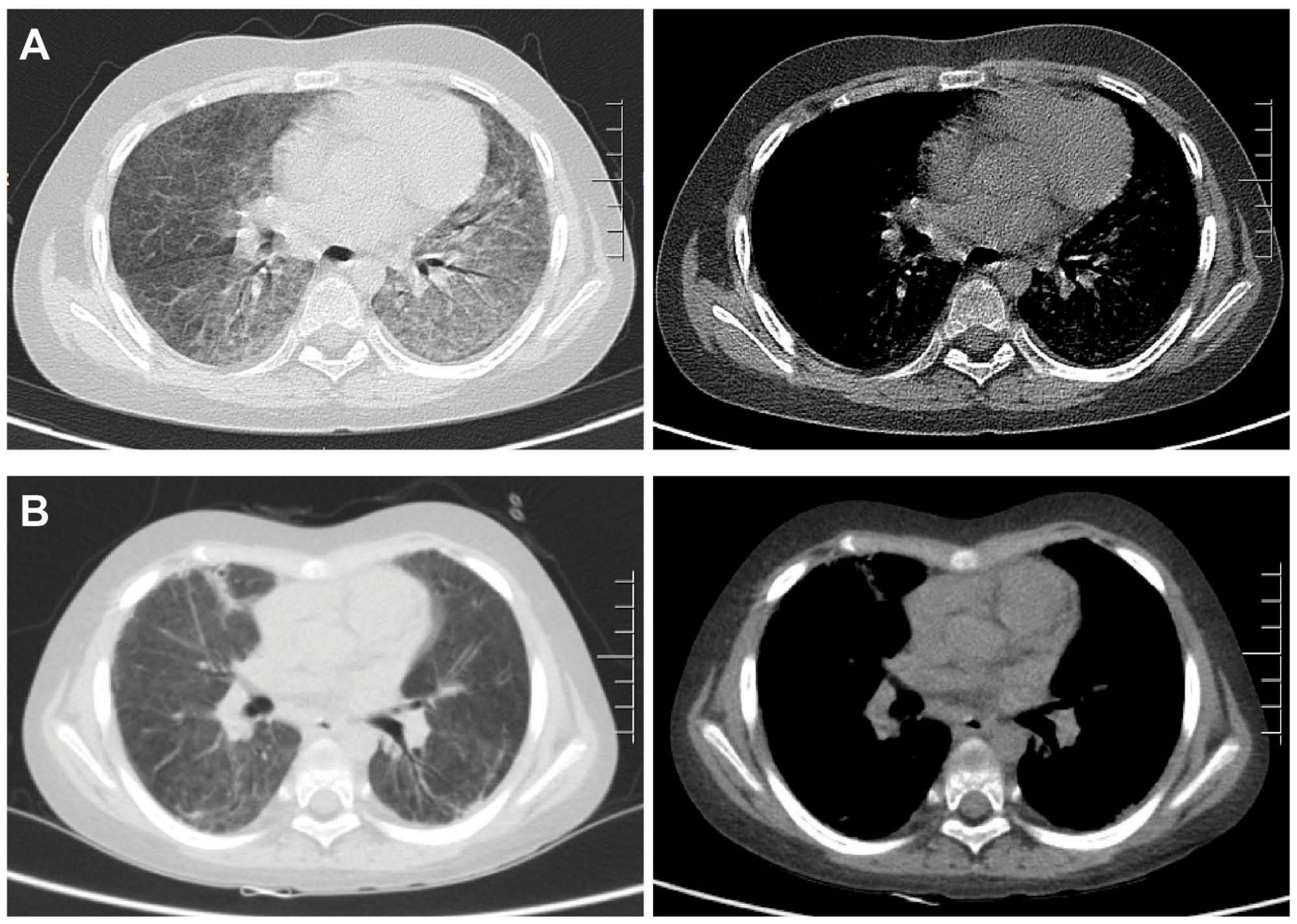

Figure I Representative chest CT images of a pediatric PCP patient (Case I3) on admission and discharge. (A) Chest CT images on admission showing diffuse ground-glass opacities in bilateral lungs. (B) Chest CT images on discharge showing that the majority of the bilateral pulmonary lesions were absorbed after anti-PCP treatment.

pathogens (Figure 2). Eight of 13 PCP patients had mixed infections of cytomegalovirus, 6 had Epstein-Barr virus, and another 6 had Streptococcus pneumoniae. Besides, Mycoplasma pneumoniae was detected by multiplex realtime PCR in 2 patients, and Candida albicans in one patient. One female patient in non-PCP group was detected as $P$. jirovecii positive by multiplex real-time PCR, and co-pathogens included Streptococcus pneumoniae and cytomegalovirus.

\section{Diagnostic Performance of Sputum} Multiplex Real-Time PCR in Pediatric PCP Serum BDG and LDH are commonly used serological markers of $\mathrm{PCP}$, and the combination of BDG testing with LDH level has been reported to significantly facilitate the diagnosis of PCP. ${ }^{7}$ Our data also showed that serum BDG and LDH levels of PCP patients were markedly higher than non-PCP group. Therefore, we further compared the diagnostic performance of multiplex realtime PCR with serum BDG plus LDH in pediatric PCP. As shown in Table 3, multiplex real-time PCR showed a trend of higher sensitivity than serum BDG plus LDH (100\% vs $76.9 \%, P=0.066$ ). Furthermore, the specificity of multiplex real-time PCR was $98.0 \%$, which was significantly better than that of BDG plus LDH (84.4\%, $P=0.021)$. Multiplex real-time PCR was also superior to serum BDG plus LDH in terms of PPV (92.9\% vs $66.7 \%, P=0.082)$ and NPV ( $100 \%$ vs $90.0 \%, P=0.024)$.

\section{Impact of Multiplex Real-Time PCR on the Modifications of Antimicrobial Agents in Pediatric PCP Patients}

Records of antimicrobial treatment during hospitalization were retrieved from 13 pediatric PCP patients. Based on the multiplex real-time PCR results, initial antimicrobial agents were changed in $76.9 \%$ of patients. Specifically, 
Table 2 Multiplex Real-Time PCR Results on Sputum of Pediatric PCP Patients

\begin{tabular}{|l|c|c|c|}
\hline $\begin{array}{l}\text { Case } \\
\text { No. }\end{array}$ & $\begin{array}{c}\text { Time from Sampling to Report } \\
\text { (Days) }\end{array}$ & $\begin{array}{c}\text { Time from Admission to Report } \\
\text { (Days) }\end{array}$ & $\begin{array}{c}\text { Pathogens Identified by Multiplex } \\
\text { PCR }\end{array}$ \\
\hline 1 & 1 & 2 & PJ, S. pneumoniae, CMV, EBV \\
2 & 1 & 2 & PJ, S. pneumoniae, M. pneumoniae, CMV \\
3 & 1 & 2 & PJ, S. pneumoniae, C. albicans, CMV \\
4 & 1 & 6 & PJ, CMV \\
5 & 1 & 2 & PJ, CMV \\
6 & 1 & 1 & PJ, S. pneumoniae \\
7 & 1 & 1 & PJ, EBV \\
8 & 1 & 1 & PJ, S. pneumoniae, CMV, EBV \\
9 & 1 & 1 & PJ \\
10 & 1 & 8 & PJ, EBV \\
11 & 1 & 1 & PJ, CMV, EBV \\
12 & 1 & 3 & PJ, S. pneumoniae \\
13 & 1 & 14 & PJ, CMV, EBV, M. pneumoniae \\
\hline
\end{tabular}

Abbreviations: PJ, pneumocystis jiroveci; CMV, cytomegalovirus; EBV, Epstein-Barr virus; S. pneumoniae, Streptococcus pneumoniae; M. pneumoniae, Mycoplasma pneumoniae; C. albicans, Candida albicans.

$23.1 \%$ of patients got 1 or 2 antimicrobial agents removed, $38.5 \%$ got antimicrobial spectrum reduced, and $38.5 \%$ got 1 or 2 antimicrobial agents added (Table 4). Remarkably, $46.2 \%$ of patients did not receive trimethoprimsulfamethoxazole of therapeutic dose until the report of multiplex real-time PCR results. All pediatric PCP patients had positive response to anti-PCP therapy.

\section{Discussion}

In the present study, we explored the diagnostic performance of multiplex real-time PCR on sputum in children with PCP. Our data showed that sputum multiplex real-time PCR had a high sensitivity and specificity for the diagnosis of PCP and was advantageous to identify co-pathogens in pulmonary mixed infections.
PCP is a serious opportunistic infection which can be life-threatening in immunocompromised hosts, ${ }^{12}$ including children and adults. It usually progresses to respiratory failure within a short time and can cause a mortality rate as high as $10-20 \%$ in HIV-infected patients and $30-60 \%$ in non-HIV infected ones. ${ }^{13}$ Compared to HIV population, mortality of non-HIV PCP patients is higher because they usually present acute onset, faster progression, and are more likely to be overlooked. So far, prognosis of PCP in pediatric population remains poor. It has been reported that the mortality rate of PCP in non-HIV children was $41.7 \%$, demonstrating that PCP is a serious infection in children, and there is an urgent need for improving prognosis. $^{3}$ Early diagnosis is crucial for prompt and appropriate treatment of PCP patients. So far, direct

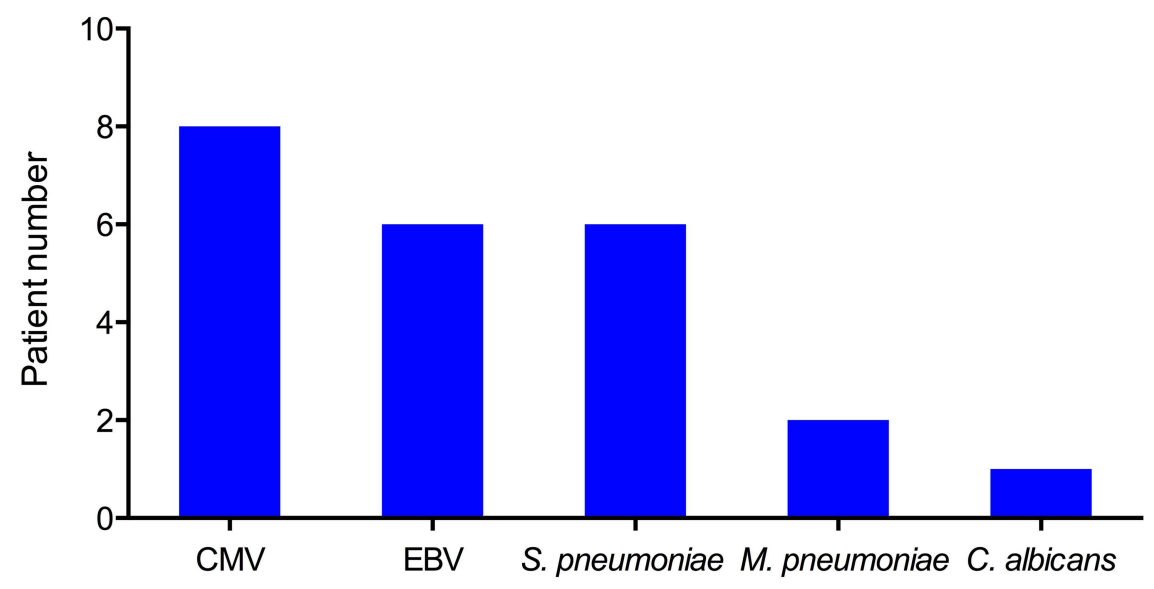

Figure 2 Number of co-pathogens identified by multiplex real-time PCR in 13 pediatric patients.

Abbreviations: CMV, cytomegalovirus; EBV, Epstein-Barr virus; S. pneumoniae, Streptococcus pneumoniae; M. pneumoniae, Mycoplasma pneumoniae; C. albicans, Candida albicans. 
Table 3 Diagnostic Performance of Multiplex Real-Time PCR and Serum BDG Plus LDH in Pediatric PCP Patients

\begin{tabular}{|c|c|c|c|c|c|c|}
\hline Methods & $\operatorname{PCP}(n=13)$ & Non-PCP $(n=50)$ & Sensitivity & Specificity & PPV & NPV \\
\hline $\begin{array}{l}\text { PCR } \\
+ \\
-\end{array}$ & $\begin{array}{c}13 \\
0\end{array}$ & $\begin{array}{c}1 \\
49\end{array}$ & $100 \%$ & $98.0 \%$ & $92.9 \%$ & $100 \%$ \\
\hline $\begin{array}{l}\mathrm{BDG}+\mathrm{LDH} \\
+ \\
-\end{array}$ & $\begin{array}{c}10 \\
3\end{array}$ & $\begin{array}{c}5 \\
27\end{array}$ & $76.9 \%$ & $84.4 \%$ & $66.7 \%$ & $90.0 \%$ \\
\hline$P$ value & & & 0.066 & 0.021 & 0.082 & 0.024 \\
\hline
\end{tabular}

Note: Serum BDG $\geq 80 \mathrm{ng} / \mathrm{L}$ and $\mathrm{LDH} \geq 250 \mathrm{U} / \mathrm{L}$ were defined as positive.

Abbreviations: BDG, (I,3)- $\beta$-D-glucan; LDH, lactate dehydrogenase; PPV, positive predict value; NPV, negative predict value.

Table 4 Impact of Multiplex Real-Time PCR on the Modifications of Antimicrobial Agents in Pediatric PCP Patients

\begin{tabular}{|l|c|}
\hline Indicators & PCP Patients (n [\%]) \\
\hline Modifications of antimicrobial agents & \\
Remove I agent & $2(15.4)$ \\
Remove 2 agents & I (7.7) \\
Reduce antimicrobial spectrum & $5(38.5)$ \\
Add I agent & $4(30.8)$ \\
Add 2 agents & $I(7.7)$ \\
Add TMP-SMZ & $6(46.2)$ \\
Add Caspofungin & $1(7.7)$ \\
Add Clindamycin & $1(7.7)$ \\
Add antiviral agent & $5(38.5)$ \\
No change & $3(23.1)$ \\
\hline Positive response to anti-PJ therapy & $13(100)$ \\
\hline
\end{tabular}

Notes: TMP-SMZ, trimethoprim-sulfamethoxazole; remove I (or 2) agent, the number of antimicrobial agent types reduced by I (or 2) after the report of mNGS results; add I (or 2) agent, the number of antimicrobial agent types reduced by I (or 2) after the report of mNGS results.

Abbreviation: PJ, pneumocystis jiroveci.

microscopic examination and PCR are widely used for the microbiological diagnosis of PCP. While positive microscopic examination is considered as the golden standard, it requires a heavy pathogen burden and experienced microbiologists to ensure the detection rate of $P$. jirovecii under microscope. Serum BDG and LDH are highly sensitive but unspecific serological biomarkers of PCP. ${ }^{4,14}$ In previous studies, PCR has been reported to be highly sensitive and specific in detecting $P$. jirovecii in respiratory tract specimen. ${ }^{15}$ PCR is also recommended as a biological diagnostic tool of PCP by clinical guidelines. ${ }^{16}$ However, it delivers little value to identify mixed infections, which are frequently observed in immunocompromised patients, by a single run of standard PCR. In the present study, we used multiplex real-time PCR in pediatric patients with presumptive PCP, which allowed rapid detection of $P$. jirovecii and other common respiratory co-pathogens. Our data showed that the sensitivity and specificity of multiplex PCR was $100 \%$ and $98.0 \%$ for the diagnosis of PCP in children, which was significantly better than the combination of serum BDG and LDH.

It is worth noting that one patient in non-PCP group was detected as $P$. jirovecii positive by multiplex real-time PCR assay. This was a two-month-old female infant without any confirmed underlying disease. She mainly presented with productive cough, bilateral patchy shadowing and consolidation of chest CT images. Peripheral white blood cell counting and serum procalcitonin level were elevated, while serum BDG was not. Multiplex PCR testing of sputum detected P. jirovecii, Streptococcus pneumoniae and cytomegalovirus. The patient got recovered after 10 days of antimicrobial treatment with Ceftriaxone, which directly supported the diagnosis of non-PCP pneumonia. Therefore, when physicians interpret the results of microbiological testing including PCR, comprehensive analysis of clinical symptoms, laboratory findings and chest radiology are necessary.

Another distinct advantage of multiplex PCR over other microbiological tests is the broad spectrum of pathogen targets, which allows the identification of mixed infections in PCP patients. Mixed infections are common in immunocompromised population, including PCP patients. ${ }^{17}$ Thus, a broad-spectrum diagnostic tool that targets various respiratory pathogens is urgently needed for comprehensive antimicrobial therapeutic strategy in PCP patients. In this study, co-pathogens were identified in 12 out of 13 patients, among which cytomegalovirus and Epstein-Barr virus were the most common ones. Furthermore, unbiased broad-spectrum detection by multiplex real-time PCR provided guidance for effective 
antimicrobial treatment. Our data showed that antimicrobial agents were changed in $76.9 \%$ of pediatric PCP patients. Nearly half $(46.2 \%)$ of patients did not receive trimethoprim-sulfamethoxazole of therapeutic dose until the report of PCR results. Additionally, multiplex realtime PCR narrowed antimicrobial spectrum in $38.5 \%$ of pediatric PCP patients, potentially reducing the abuse of antimicrobial agents. These data indicate that multiplex real-time PCR facilitates the proper antimicrobial treatment in children with PCP.

BALF is usually considered as a lower respiratory tract specimen superior to sputum for defining pulmonary microbiology, but it has important limitations. Bronchoalveolar lavage is invasive, time-consuming and usually costs hundreds of dollars. For many pediatric patients who cannot act as bronchoscopists required, general anesthesia and moderate-to-deep sedation are needed, which directly increases anesthetic risk. Anesthetic risk can be even higher for PCP patients due to severe hypoxemia. On the contrary, sputum is non-invasive, readily obtained, repeatable, and requires no anesthesia. During the past decades, sputum is widely used in microscopic examination, culture and molecular biological testing (including PCR) for the diagnosis of pulmonary infectious diseases. Induced sputum was reported to be a sensitive and cost-effective respiratory tract specimen for HIVinfected PCP patients. ${ }^{18,19}$ In 1998, Caliendo and his colleagues found that the sensitivity and specificity of PCR assay on induced sputum for the detection of $P$. jirovecii was $94 \%$ and $90 \%$, respectively. ${ }^{20}$ Afterwards, Pinlaor et al also reported that PCR on sputum samples reached a high sensitivity of $84.62 \%$ and specificity of $98.4 \%$ for the diagnosis of PCP in immunocompromised adults. ${ }^{10}$ However, diagnostic performance of sputum in pediatric PCP patients has been unexplored. In this study, our results showed that multiplex real-time PCR on sputum had a good performance in children with PCP, suggesting that sputum may be an alternative to BALF in pediatric patients, especially for those who either could not tolerate or declined bronchoscope examination.

This study has limitations. First of all, it is a singlecenter retrospective study with a relatively small case number, thus intrinsic bias was unavoidable. Second, comparison of PCR assay between matched sputum and BALF samples was not performed, because bronchoscopic examination was considered as infeasible or unsafe for many pediatric patients in this study. Finally, multiplex real-time PCR on sputum has drawbacks, while it offers advantages.
It is difficult to distinguish $P$. jirovecii colonization from infection because there are no widely accepted quantitative cutoffs or threshold values for quantitative PCR. Therefore, definite diagnosis of PCP must be made based on the comprehensive analysis of clinical characteristics, laboratory abnormalities, radiological findings and microbiological testing, rather than PCR results alone.

\section{Conclusions}

Multiplex real-time PCR on sputum is a useful diagnostic tool for pediatric PCP patients. Sputum sampling is simple, non-invasive and cheap. Multiplex real-time PCR has a high sensitivity and specificity in identifying $P$. jirovecii and is advantageous in identifying mixed infections. Multiplex real-time PCR on sputum is suggested in pediatric patients with presumptive diagnosis of PCP, especially for those who either cannot tolerate or decline bronchoscope examination.

\section{Acknowledgment}

The authors appreciate the professionalism and compassion demonstrated by all the healthcare workers involved in patient care. We acknowledge all the patients for their involvement in this study.

\section{Funding}

This work was supported by the Youth Research Foundation of Xiangya Hospital (grant number 2018Q015).

\section{Disclosure}

The authors declare that they have no conflicts of interest.

\section{References}

1. Morris A, Lundgren JD, Masur H, et al. Current epidemiology of Pneumocystis pneumonia. Emerg Infect Dis. 2004;10(10):1713-1720. doi:10.3201/eid1010.030985

2. Garcia-Moreno J, Melendo-Perez S, Martin-Gomez MT, et al. Pneumocystis jirovecii pneumonia in children. A retrospective study in a single center over three decades. Enferm Infecc Microbiol Clin. 2020;38(3):111-118. doi:10.1016/j.eimc.2019.05.005

3. Ling C, Qian S, Wang Q, et al. Pneumocystis pneumonia in non-HIV children: a 10-year retrospective study. Clin Respir J. 2018;12 (1):16-22. doi:10.1111/crj.12467

4. Del Corpo O, Butler-Laporte G, Sheppard DC, Cheng MP, McDonald EG, Lee TC. Diagnostic accuracy of serum (1-3)-beta -D-glucan for Pneumocystis jirovecii pneumonia: a systematic review and meta-analysis. Clin Microbiol Infect. 2020;26(9):1137-1143. doi:10.1016/j.cmi.2020.05.024

5. Vogel M, Weissgerber P, Goeppert B, et al. Accuracy of serum LDH elevation for the diagnosis of Pneumocystis jiroveci pneumonia. Swiss Med Wkly. 2011;141:w13184. 
6. Procop GW, Haddad S, Quinn J, et al. Detection of Pneumocystis jiroveci in respiratory specimens by four staining methods. J Clin Microbiol. 2004;42(7):3333-3335. doi:10.1128/JCM.42.7.33333335.2004

7. White PL, Backx M, Barnes RA. Diagnosis and management of Pneumocystis jirovecii infection. Expert Rev Anti Infect Ther. 2017;15(5):435-447. doi:10.1080/14787210.2017.1305887

8. Huang HS, Tsai CL, Chang J, Hsu TC, Lin S, Lee CC. Multiplex PCR system for the rapid diagnosis of respiratory virus infection: systematic review and meta-analysis. Clin Microbiol Infect. 2018;24 (10):1055-1063. doi:10.1016/j.cmi.2017.11.018

9. Lee SH, Ruan SY, Pan SC, Lee TF, Chien JY, Hsueh PR. Performance of a multiplex PCR pneumonia panel for the identification of respiratory pathogens and the main determinants of resistance from the lower respiratory tract specimens of adult patients in intensive care units. J Microbiol Immunol Infect. 2019;52(6):920-928. doi:10.1016/j.jmii.2019.10.009

10. Pinlaor S, Mootsikapun P, Pinlaor P, et al. PCR diagnosis of Pneumocystis carinii on sputum and bronchoalveolar lavage samples in immuno-compromised patients. Parasitol Res. 2004;94 (3):213-218. doi:10.1007/s00436-004-1200-y

11. Pennington K, Wilson J, Limper AH, Escalante P. Positive Pneumocystis jirovecii Sputum PCR results with negative bronchoscopic PCR results in suspected Pneumocystis pneumonia. Can Respir J. 2018;2018:6283935.

12. Gaborit BJ, Tessoulin B, Lavergne RA, et al. Outcome and prognostic factors of Pneumocystis jirovecii pneumonia in immunocompromised adults: a prospective observational study. Ann Intensive Care. 2019;9(1):131. doi:10.1186/s13613-019-0604-x

13. Cilloniz C, Dominedo C, Alvarez-Martinez MJ, et al. Pneumocystis pneumonia in the twenty-first century: HIV-infected versus HIV-uninfected patients. Expert Rev Anti Infect Ther. 2019;17 (10):787-801. doi:10.1080/14787210.2019.1671823
14. Salzer HJF, Schafer G, Hoenigl M, et al. Clinical, diagnostic, and treatment disparities between HIV-infected and non-HIV-infected immunocompromised patients with Pneumocystis jirovecii Pneumonia. Respiration. 2018;96(1):52-65. doi:10.1159/000487713

15. Guegan H, Robert-Gangneux F. Molecular diagnosis of Pneumocystis pneumonia in immunocompromised patients. Curr Opin Infect Dis. 2019;32(4):314-321. doi:10.1097/QCO.0000000000000559

16. Alanio A, Hauser PM, Lagrou K, et al. ECIL guidelines for the diagnosis of Pneumocystis jirovecii pneumonia in patients with haematological malignancies and stem cell transplant recipients. J Antimicrob Chemother. 2016;71(9):2386-2396. doi:10.1093/jac/ dkw156

17. Peng JM, Du B, Qin HY, Wang Q, Shi Y. Metagenomic next-generation sequencing for the diagnosis of suspected pneumonia in immunocompromised patients. $J$ Infect. 2021;82(4):22-27. doi:10.1016/j.jinf.2021.01.029

18. Turner D, Schwarz Y, Yust I. Induced sputum for diagnosing Pneumocystis carinii pneumonia in HIV patients: new data, new issues. Eur Respir J. 2003;21(2):204-208. doi:10.1183/ 09031936.03 .00035303

19. Cruciani M, Marcati P, Malena M, Bosco O, Serpelloni G, Mengoli C. Meta-analysis of diagnostic procedures for Pneumocystis carinii pneumonia in HIV-1-infected patients. Eur Respir J. 2002;20(4):982-989. doi:10.1183/09031936.02.01372002

20. Caliendo AM, Hewitt PL, Allega JM, Keen A, Ruoff KL, Ferraro MJ. Performance of a PCR assay for detection of Pneumocystis carinii from respiratory specimens. J Clin Microbiol. 1998;36(4):979-982. doi:10.1128/JCM.36.4.979-982.1998
Infection and Drug Resistance

\section{Publish your work in this journal}

Infection and Drug Resistance is an international, peer-reviewed openaccess journal that focuses on the optimal treatment of infection (bacterial, fungal and viral) and the development and institution of preventive strategies to minimize the development and spread of resistance. The journal is specifically concerned with the epidemiology of
Dovepress

antibiotic resistance and the mechanisms of resistance development and diffusion in both hospitals and the community. The manuscript management system is completely online and includes a very quick and fair peerreview system, which is all easy to use. Visit http://www.dovepress.com/ testimonials.php to read real quotes from published authors. 